\title{
Pathological Significance and Prognostic Roles of Thrombospondin-3, 4 and 5 in Bladder Cancer
}

\author{
JUNKI HARADA, YASUYOSHI MIYATA, KYOHEI ARAKI, TSUYOSHI MATSUDA, \\ YOSHIAKI NAGASHIMA, YUTA MUKAE, KENSUKE MISTUNARI, \\ TOMOHIRO MATSUO, KOJIRO OHBA, YASUSHI MOCHIZUKI and HIDEKI SAKAI
}

Department of Urology, Nagasaki University Graduate School of Biomedical Sciences, Nagasaki, Japan

\begin{abstract}
Background/Aim: The pathological significance of thrombospondin (TSP)-1 and -2 in bladder cancer $(B C)$ is well-known whereas that of TSP-3, 4 and 5 remains unclear. Our aim is to clarify the pathological significance and prognostic roles of TSP-3 to 5 expression in BC patients. Patients and Methods: TSP-3 to 5 expression, proliferation index (PI), apoptotic index (AI) and microvessel density (MVD) were evaluated in 206 BC patients by immunohistochemical techniques. Results: TSP-5 expression was positively associated with grade, $T$ stage, metastasis, and worse prognosis. PI in TSP-5-positive tissues was significantly higher compared to negative tissues. In contrast, AI in TSP-5-positive tissues was significantly lower compared to negative tissues. Expressions of TSP-3 and 4 were not associated with any clinicopathological features, survival, PI, or AI. Conclusion: TSP-5 plays important roles in malignant behavior via cell survival regulation whereas the pathological significance of TSP-3 and TSP-4 in BC might be minimal.
\end{abstract}

Bladder cancer (BC) is the most common urinary system malignancy and is increasing in developed countries (1). Urothelial carcinoma (UC), arising from the urothelium, is the most common histological type of BC. Depending on whether BC invades the muscle layers of the urinary bladder, $\mathrm{BC}$ is divided into two groups: non-muscle invasive bladder cancer (NMIBC) and muscle invasive bladder cancer (MIBC).

This article is freely accessible online.

Correspondence to: Yasuyoshi Miyata, Department of Urology, Nagasaki University Graduate School of Biomedical Sciences, 1-71 Sakamoto, Nagasaki 852-8501, Japan. Tel: +81 958197340, Fax: +81 958197343, e-mail: yasu-myt@ nagasaki-u.ac.jp

Key Words: TSPs, pathological features, survival, cell proliferation, apoptosis, bladder cancer.
The standard treatment strategy for NMIBC differs considerably from that for MIBC. In short, NMIBC is mainly treated with transurethral resection, whereas MIBC is treated with radical cystectomy (1). However, pathological features including grade, tumor growth, invasion, and metastasis are closely associated with prognosis in both NMIBC and MIBC. Furthermore, cancer-related characteristics such as cancer cell proliferation, apoptosis, and angiogenesis, play crucial roles in malignant aggressiveness (2-4). Thus, understanding the pathological mechanisms of such tumor growth and progression is essential for designing treatment and monitoring strategies for patients with BC. The molecular and genetic abnormalities in BC have been investigated, and novel cancer-related molecules and predictive markers have been reported (5-7). However, more detailed information is required about the molecular mechanisms of malignant aggressiveness in BC because the outcomes including survival and recurrence in these patients remain unsatisfactory.

Thrombospondins (TSPs) are extracellular glycoproteins and are divided into two sub-groups based on their domain structure: sub-group A (TSP-1 and TSP-2 form trimers) and sub-group B [TSP-3, TSP-4, and TSP-5 (also called cartilage oligomeric matrix protein; COMP) form pentamers] (8). One of the most common biological roles of TSPs is tissue remodeling via interaction with the extracellular matrix under physiological and pathological conditions, including cancers $(9,10)$. The pathological roles of TSP-1 and TSP-2 are well studied and a large amount of evidence has been accumulated regarding their roles in many types of malignancies, including BC (11-17). In contrast, despite similar studies about TSP-3 to $-5(18,19)$, the available information is overwhelmingly lacking, when compared to TSP-1 and 2. In particular, only a few studies have explored the pathological significance and predictive roles of TSP-3 to 5 in patients with $\mathrm{BC}$.

In recent years, many investigators have focused on the tumor microenvironment in accordance with the development of new immune therapy and molecular-targeted 
therapy; TSPs are well-known as tumor microenvironment modulators in various types of malignancies, including BC $(10,20)$. Furthermore, interactions between TSP sub-groups $\mathrm{A}$ and $\mathrm{B}$ have been reported in various biological functions $(21,22)$. Therefore, understanding the pathological significance of TSP sub-group B at the clinical and molecular levels is important for the efficient monitoring and treatment of patients with BC. The main aim of this study was to clarify the relationships of TSP-3 to 5 expression with clinicopathological features, including grade, tumor growth, and metastasis, in patients with $\mathrm{BC}$. We also investigated the proliferative, apoptotic, and angiogenic activities of these TSPs and performed survival analyses in the patients.

\section{Patients and Methods}

Patients. We investigated the expression of TSP-3, 4 and 5 in 206 patients with $\mathrm{BC}$, including 150 with NMIBC and 46 with MIBC. In addition to cancer tissues, 20 normal urothelial tissues were also examined. All included patients were diagnosed with urothelial cancer of the bladder by routine pathological diagnosis at Nagasaki University Hospital, Nagasaki, Japan. To determine the presence of metastasis, all patients with MIBC underwent magnetic resonance imaging of the urinary bladder, computed tomography of the abdomen and pelvis, bone scanning, and lung X-ray imaging. The $\mathrm{T}$ stage at diagnosis was categorized into two subgroups, the NMIBC group (Ta-T1) and MIBC group (T2-T4).

This retrospective study complied with the principles of the Declaration of Helsinki and its revisions. This study was approved by the institutional review board of Nagasaki University Hospital (No: 12052899), and all participants provided written informed consent.

Immunohistochemistry. Formalin-fixed sections (5- $\mu$ m-thick) were deparaffinized and rehydrated, and all antigens were retrieved using $0.01 \mathrm{M}$ sodium citrate buffer. All sections were immersed in hydrogen peroxide to block endogenous peroxidase, and were subsequently probed overnight at $4^{\circ} \mathrm{C}$ with the primary antibodies. Primary antibodies were obtained from Santa Cruz Biotechnology Inc. (Santa Cruz, CA, USA; against TSP-3, sc-25348 and TSP-4, sc-28293), Lifespan BioSciences, Inc. (Seatle, WA, USA; against COMP/TSP-5, LS-B12308), DakoCytomation (Glostrup, Denmark; against Ki-67, M7240 and CD34, M7165), and R\&D systems Inc. (Abingdon, UK; against cleaved caspase-3, MAB835). Sections were then labeled with peroxidase using Dako EnVision $+^{\mathrm{TM}}$ Peroxidase (Dako, Carpinteria, CA, USA). The peroxidase reaction was visualized using a 3,3'-diaminobenzidine tetrahydrochloride substrate kit (Zymed Laboratories Inc., San Francisco, CA, USA). Finally, the sections were counterstained using hematoxylin.

For the expression of TSP-3 to 5, immunoreactivity was scored from 0 to 4 according to previous reports $(23,24)$, and scores of 3 and 4 were judged as positive expression. Such evaluation was performed at 5-10 randomly chosen fields, with at least 500 cancer cells. To determine microvessel density (MVD), CD34 stained vessels were measured at 3-5 "hot spots;" MVD was defined as the number of stained vessels per field at $200 \times$ magnification. Proliferation index (PI) and apoptotic index (AI) were determined by the percentage of cells stained with anti-Ki-67 and anti-cleaved caspase- 3 antibodies, respectively. Slides were examined using a computer-aided image analysis system (Win ROOF version 5.0; MITANI, Fukui, Japan), and were scored twice at different times by TM and YM, who were blinded to the clinicopathological and survival data. Detailed methods of the immunohistochemical techniques, including negative and positive controls, have been described in our previous reports $(2,11,25,26)$.

Statistical analyses. The Student' s $t$-test was used to compare continuous variables. Categorical variables were compared using the chi-squared test. Kaplan-Meier curves and log rank tests were used for survival analyses. All statistical analyses were two-sided, and significance was defined at $p<0.05$.

\section{Results}

Expression of TSP-3, TSP4 and TSP-5 in bladder cancer tissues. The representative expression of TSP-3, TSP-4, and TSP-5 in BC tissues is shown in Figure 1A, B, and C, respectively. In cancer cells, TSP-3 to 5 were mainly detected in the cytoplasm. Moderate to strong expression of TSP-3 and 5 was rare in stromal and connective tissue cells. Therefore, in this study, we analyzed their cytoplasmic expression. In TSP-4 and 5, strong expression was relatively rare compared to TSP-5. In addition, in TSP-5, strongly stained cancer cells were often detected in invasive BC cells (Figure 1D and E), such finding was not found in TSP-3 and -4. Finally, 12 (5.8\%), 88 (42.7\%), and 119 (57.8\%) patients were judged to have positive expression of TSP-3, 4 and 5, respectively. In contrast, moderate to strong expression of TSP-3 to 5 was not observed in the 20 normal urothelial tissues; thus, all normal tissues were judged to have negative expression of TSP-3 to 5 .

Correlation with clinicopathological features. The relationship between TSP-3 to TSP-5 expression and clinicopathological features is shown in Table I. TSP-3 and TSP-4 showed no significant association with any of the parameters including grade, $\mathrm{T}$ stage, muscle invasion, and metastasis. In contrast, as shown in Table I, the positively stained ratio of TSP-5 in high-grade disease $(65.5 \%)$ was significantly higher $(p=0.013)$ than that in low-grade disease (48.3\%). Similarly, TSP-5 expression was positively associated with high $\mathrm{T}$ stage $(p=0.012)$ and presence of metastasis $(p=0.002)$. In addition, TSP-5 expression was significantly associated with invasion into the muscle layer $(p=0.019)$ and the lamina propria $(p=0.019)$ (Table I).

Survival analyses. The Kaplan-Meier survival curves for cause-specific survival are shown in Figure 2. Positive expression of TSP-3 tended to have poor prognosis; however, this difference was not significant ( $p=0.342$; Figure 2A). Likewise, the survival curve of patients with TSP-4positive BC patients was similar to that in patients with TSP- 

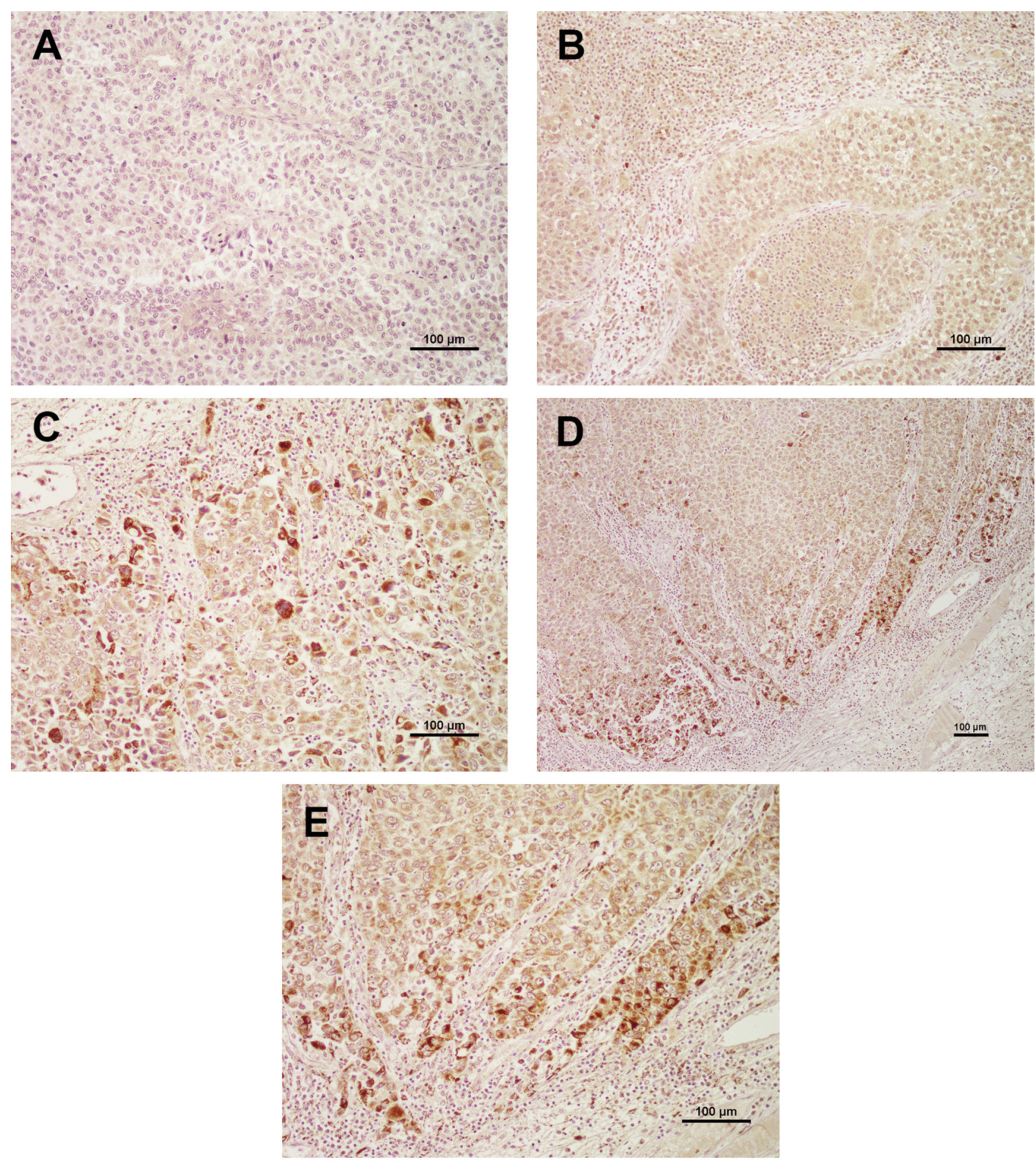

Figure 1. Representative expression of TSP-3 (A), TSP-4 (B), and TSP-5 (C) (all magnifications are $\times 200$ ). In TSP-5, strongly stained cells were often detected in the invasive front (D: magnification is $\times 100, E: \times 200)$.

4-negative BC ( $p=0.969$; Figure $2 \mathrm{~B})$. In contrast, the survival rate of patients positively expressing TSP-5 was significantly worse than that of the TSP-5-negative patients $(p=0.023$; Figure 2C).
Correlation with cancer cell proliferation, apoptosis, and angiogenesis. The relationships of TSP-3 to 5 expression with PI, AI, and MVD are shown in Figure 3. The mean/SD of PIs in TSP-3-positive tissues (27.5/9.7\%) showed no 
in vivo $35: 1693-1701(2021)$

Table I. Relationships between thrombospondin-3 to 5 and clinicopathological features.

\begin{tabular}{|c|c|c|c|c|c|c|}
\hline \multirow[b]{2}{*}{ Predictor } & \multicolumn{2}{|c|}{ Thrombospondin-3 } & \multicolumn{2}{|c|}{ Thrombospondin-4 } & \multicolumn{2}{|c|}{ Thrombospondin-5 } \\
\hline & Negative & Positive & Negative & Positive & Negative & Positive \\
\hline \multicolumn{7}{|l|}{ Age } \\
\hline Mean & 69.3 & 70.3 & 70.2 & 68.3 & 69.0 & 69.4 \\
\hline SD & 12.0 & 10.4 & 11.1 & 12.8 & 12.3 & 11.6 \\
\hline$p$-Value & \multicolumn{2}{|c|}{0.775} & \multicolumn{2}{|c|}{0.269} & \multicolumn{2}{|c|}{0.708} \\
\hline \multicolumn{7}{|c|}{ Gender; N/\% } \\
\hline Male & $151 / 77.8$ & $11 / 22.2$ & $95 / 58.6$ & $67 / 41.7$ & $68 / 42.0$ & $94 / 68.0$ \\
\hline Female & $43 / 91.7$ & $1 / 8.3$ & $23 / 52.3$ & $21 / 47.7$ & $18 / 40.9$ & $26 / 59.1$ \\
\hline$p$-Value & \multicolumn{2}{|c|}{0.257} & \multicolumn{2}{|c|}{0.449} & \multicolumn{2}{|c|}{0.899} \\
\hline \multicolumn{7}{|l|}{ Grade } \\
\hline Low & $84 / 96.6$ & $3 / 3.4$ & $48 / 55.2$ & $39 / 44.8$ & $45 / 51.7$ & $42 / 48.3$ \\
\hline High & $110 / 92.4$ & $9 / 7.6$ & $70 / 58.8$ & $49 / 41.2$ & $41 / 34.5$ & $78 / 65.5$ \\
\hline$p$-Value & \multicolumn{2}{|c|}{0.200} & \multicolumn{2}{|c|}{0.601} & \multicolumn{2}{|c|}{0.013} \\
\hline \multicolumn{7}{|l|}{$\mathrm{T}$ stage } \\
\hline $\mathrm{Ta}$ & $58 / 96.7$ & $2 / 3.3$ & $32 / 53.3$ & $28 / 46.7$ & $35 / 58.3$ & $25 / 41.7$ \\
\hline $\mathrm{T} 1$ & $83 / 92.2$ & $7 / 7.8$ & $51 / 56.7$ & $39 / 43.3$ & $35 / 38.9$ & $55 / 61.1$ \\
\hline $\mathrm{T} 2$ & $27 / 96.4$ & $1 / 3.6$ & $18 / 64.3$ & $10 / 35.7$ & $9 / 32.1$ & $19 / 67.9$ \\
\hline $\mathrm{T} 3$ & $16 / 88.9$ & $2 / 11.1$ & $13 / 72.2$ & $5 / 27.8$ & $6 / 33.3$ & $12 / 66.7$ \\
\hline $\mathrm{T} 4$ & $10 / 100$ & $0 / 0$ & $4 / 40.0$ & $6 / 60.0$ & $1 / 10.0$ & $9 / 90.0$ \\
\hline$p$-Value & \multicolumn{2}{|c|}{0.541} & \multicolumn{2}{|c|}{0.431} & \multicolumn{2}{|c|}{0.012} \\
\hline \multicolumn{7}{|l|}{ Metastasis } \\
\hline Absence & $174 / 94.1$ & $11 / 5.9$ & $104 / 56.2$ & $81 / 43.8$ & $84 / 45.5$ & $101 / 54.6$ \\
\hline Presence & $20 / 95.2$ & $1 / 4.8$ & $14 / 66.7$ & $7 / 33.3$ & 2/9.5 & $19 / 90.5$ \\
\hline$p$-Value & \multicolumn{2}{|c|}{0.826} & \multicolumn{2}{|c|}{0.359} & \multicolumn{2}{|c|}{0.002} \\
\hline \multicolumn{7}{|l|}{ Invasion } \\
\hline \multicolumn{7}{|l|}{ Muscle } \\
\hline $\mathrm{Ta} / 1$ & $141 / 94.0$ & $9 / 6.0$ & $83 / 55.3$ & $67 / 44.7$ & $70 / 46.7$ & $80 / 53.3$ \\
\hline $\mathrm{T} 2-4$ & $53 / 94.6$ & $3 / 5.4$ & $35 / 62.5$ & $21 / 37.5$ & $16 / 28.6$ & $40 / 71.4$ \\
\hline$p$-Value & 0.861 & 0.355 & 0.019 & & & \\
\hline \multicolumn{7}{|l|}{ Ta $v s . \mathrm{T} 1$} \\
\hline$p$-Value & \multicolumn{2}{|c|}{0.262} & \multicolumn{2}{|c|}{0.688} & \multicolumn{2}{|c|}{0.019} \\
\hline
\end{tabular}

significant difference $(p=0.145)$ compared to those in the negative tissues $(23.8 / 8.4 \%)$; similar results were obtained with TSP-4 expression (24.0/8.3 vs 24.0/8.0; $p=0.944$; Figure $3 \mathrm{~A})$. On the contrary, the mean/SD of PIs in TSP-5-positive tissues $(27.2 / 7.7 \%)$ was significantly higher $(p<0.001)$ than that in the TSP-5-negative tissues (19.6/7.6\%; Figure 3A). The mean/SD of AI also showed significant and negative correlations with TSP-5 expression (positive: $4.0 / 1.5 \% \mathrm{vs}$. negative: $4.7 / 2.0 \%, p=0.016$ ), but not with TSP-3 or TSP-4 ( $p=0.281$ or 0.781 , respectively; Figure $3 \mathrm{~B})$. On the contrary, as shown in Figure 3C, there was no significant correlation between MVD and TSP-3 to TSP-5 expression ( $p=0.649$, 0.711 , and 0.627 , respectively).

\section{Discussion}

In the present study, we demonstrated that TSP-5 expression was closely associated with grade, tumor growth, cancer cell progression, and survival in patients with BC, whereas TSP-3 and TSP-4 expression was not. In addition, we noticed that BC cells at the invasive front showed strong expression of TSP-5. Unfortunately, there have been no reports on the pathological significance of TSP-5 expression in human BC tissues. However, TSP-5 has been reported to play crucial roles in cell differentiation, tumor growth, invasion, and metastasis in various types of cancers $(23,24,27-30)$. For example, TSP-5 expression was significantly associated with Gleason score, $\mathrm{T}$ stage, and seminal vesicle invasion in patients with prostate cancer (24). The same study group also showed that TSP-5 expression in breast cancer cells was positively associated with lymph node metastasis and poor survival in the same patients (23). Furthermore, several other investigators have demonstrated that TSP-5 expression is positively related to cell differentiation, $\mathrm{T}$ stage, $\mathrm{N}$ stage, $\mathrm{M}$ stage, and poor outcomes including disease-free and overall survival in colorectal cancer (29-31). From these reports, our results suggest that TSP-5 plays important roles in malignant behavior and that its expression is a useful predictive marker for survival in patients with BC. 

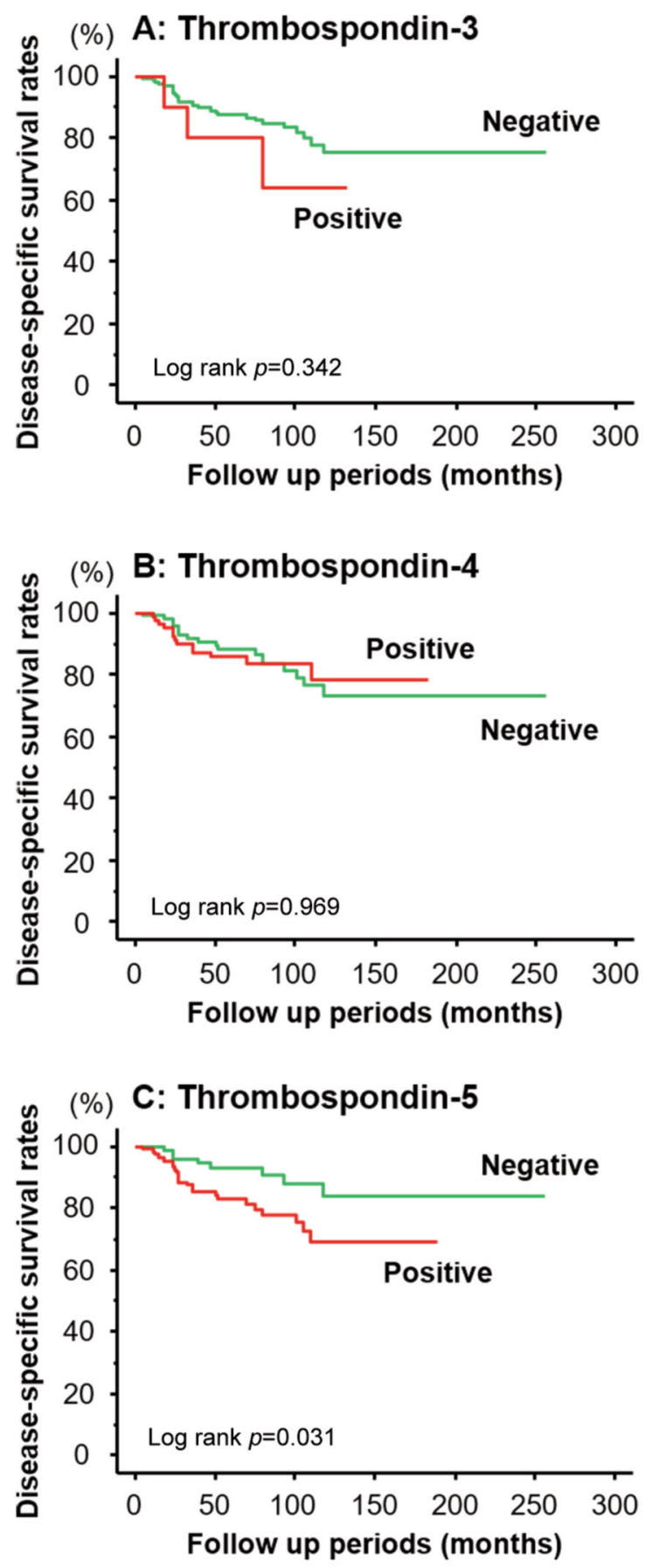

Figure 2. Kaplan-Meier survival curves for cause-specific survival and TSP-3 (A), TSP-4 (B), and TSP-5 (C) expression. Among TSP-3 to 5, patients with TSP-5-positive tissues showed worse survival compared to those with TSP-5 negative tissues $(C)$.

Stimulation of cancer cell proliferation and suppression of apoptosis were suggested as the molecular mechanisms underlying the TSP-5-induced pathological activities in BC tissues. The status of cell survival modulated by cell proliferation and apoptosis is considered a strong determinant of malignant aggressiveness in many types of cancers, including BC $(2,4,32,33)$. The pathological roles of TSP-5 expression in patients with $\mathrm{BC}$ have not been explored. However, TSP-5 is reported to promote cell proliferation in colon cancer cell lines and to suppress apoptosis in prostate cancer cell lines $(24,29)$. In addition to such in vitro studies, TSP-5 expression was positively correlated with tumor volume in the xenograft animal models of several cancers including colon cancer, breast cancer, and prostate cancer, and in the regulation of PI (measured by anti-Ki-67 antibody) (23, $24,29)$. Thus, the previous in vitro studies and animal experiments supported our results on the proliferative and anti-apoptotic activities of TSP-5 in patients with BC. However, further detailed analysis is essential to determine the proliferative and apoptotic effects of TSP-5 in BC.

Like TSP-5, TSP-4 is reported to be associated with malignant potential, tumor growth, and progression in several cancers including gastric cancer, hepatocellular carcinoma, and gallbladder cancer (34-37). However, our results showed no significant pathological role of TSP-4 expression in BC tissues. Unfortunately, the reasons underlying this discrepancy between $\mathrm{BC}$ and other cancers are unknown because there are no in vivo and in vitro studies on the pathological significance and prognostic role of TSP4 in BC. However, TSP-4 immunoreactivity was mainly detected in the "cancer cells" of BC in the present study, whereas in previous studies, TSP-4 expression was evaluated in "stromal cells" of tumor tissues or without distinguishing between "cancer cells" and "stromal cells" (34, 36-38). Therefore, we first speculated that the pathological activities of TSP-4 might originate from stromal cell expression, but not from cancer cells. In fact, TSP-4 expression in cancerrelated stromal tissues was reported to be significantly higher than that in normal stroma, normal epithelium, and cancer cells in breast cancer (39). However, an in vitro study showed that TSP-4 overexpression in colorectal cancer cell lines significantly decreased colony formation (40). Further, TSP-4 knockdown inhibited cell migration and invasion of hepatocellular cancer cell lines (41). Overall, these studies suggest that TSP-4 expression in cancer cells plays a significant role in tumor growth and cancer cell progression. However, there are contrasting opinions regarding whether TSP-4 acts as a tumor-promoter or suppressor, based on different types of malignancies (34-37, 39-41). Finally, we emphasize that further detailed studies using cancer cell lines, animal models, and clinicopathological data are necessary to discuss and conclude the clinical significance and pathological roles of TSP-4 in patients with BC.

In the present study, BC cells with moderate to strong TSP-3 expression were rare, and its expression was not associated with any of the clinicopathological features or survival. A previous report showed that TSP-3 gene 

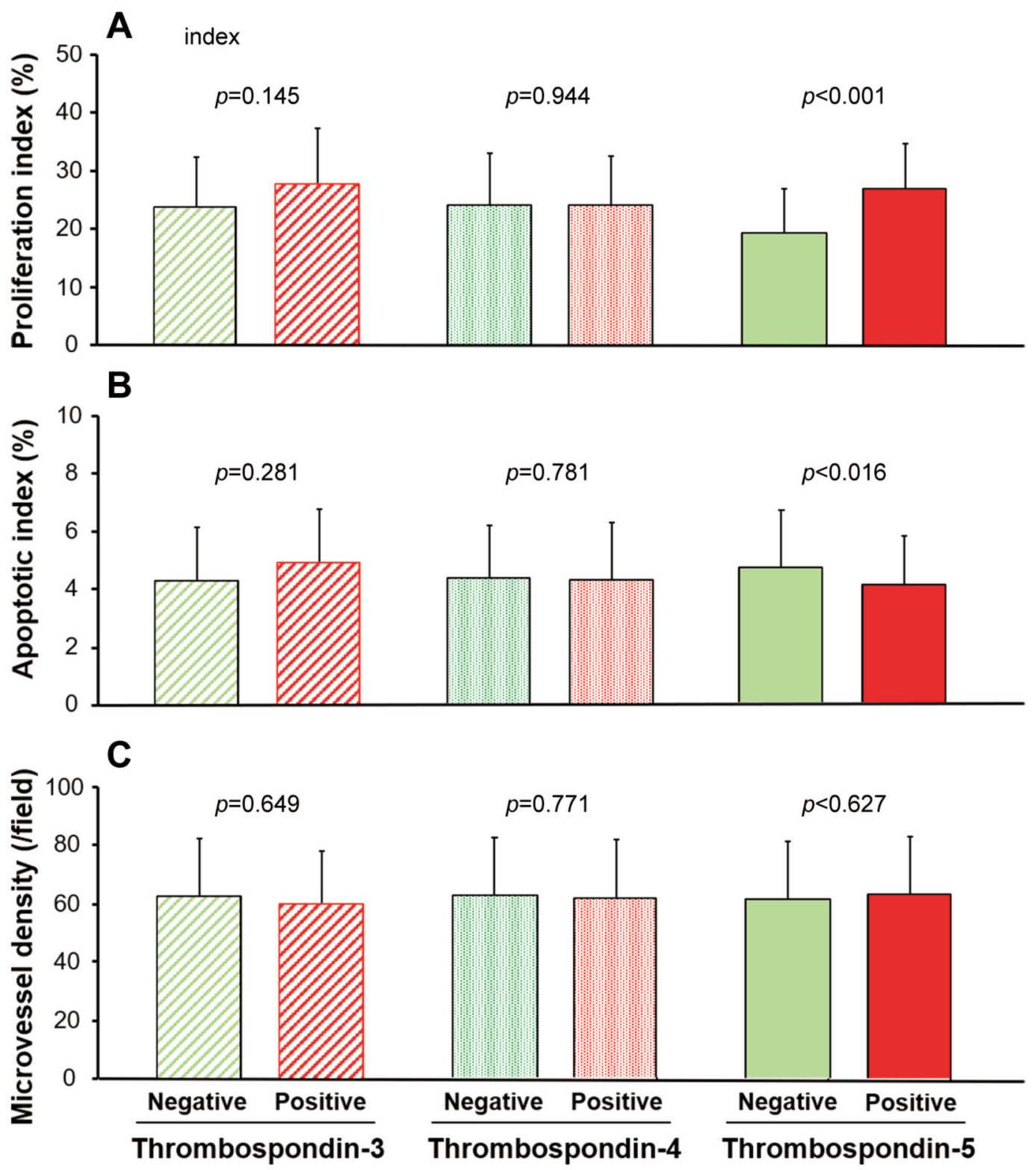

Figure 3. Proliferation index (PI; A), apoptotic index (AI; B), and microvessel density $(M V D ; C)$ are shown. Regarding PI and AI, a significant difference was observed according to the TSP-5 expression status, but not with TSP-3 and 4 (A and B). However, MVD was similar between the negative and positive expression of TSP-3 to 5.

expression was elevated in osteosarcoma tissues with metastasis, and it was identified as a useful predictor for poor survival in these patients (42). Further, the authors suggested that the TSP-3 gene acted as a stimulator of tumor progression (42). However, other investigators showed that TSP-3 expression was upregulated by NS398, a non-steroidal anti-inflammatory drug (NSAID), in lung cancer cells (43). In general, NSAIDs are considered to be tumor-suppressors in many types of malignancies (44). TSP-3 has also been described as a tumor suppressor (43). To our knowledge, other than these two findings, no information is available regarding the pathological roles of TSP-3 expression in malignancies including BC.

Contrary to our expectations, the expression of TSP-3, TSP4, and TSP-5 was not associated with MVD in BC tissues. TSP-3, TSP-4, and TSP-5 are part of the same family including TSP-1 and TSP-2, which are well-known for their antiangiogenic activities under numerous physiological and pathological conditions, including cancers $(45,46)$. Therefore, we hypothesized that TSP-3, TSP-4, and TSP-5 are closely 
associated with tumor growth via regulation of angiogenesis in $\mathrm{BC}$ tissues; however, no significant angiogenesis-related activity was detected in this study. TSP-3 gene expression has been reported to play important roles in tumor growth via maintenance of angiogenic activity in osteosarcoma (42). On the contrary, TSP-3 is also reported to have no significant inhibitory effects on angiogenesis in human dermal microvascular endothelial cells (HMVEC) (47). In addition, in pituitary tumors, TSP-3 demonstrated a minimal role in angiogenic processes, unlike that of TSP-1 and TSP-2 (48). Based on these reports and our results, we speculated that TSP3 does not play a significant role in the angiogenesis in BC tissues. However, several investigators have reported that TSP4 exerts pro-angiogenic activities in tissue remodeling and tendinopathy development $(49,50)$. Similar pro-angiogenic activities of TSP-4 have been reported through in vivo and in vitro studies on breast cancer and colorectal cancer $(51,52)$.

One of the major limitations of our study is the lack of in vitro data to confirm the results of our in vivo study. Initially, we aimed to investigate the interactions between TSP-3 to 5 in cancer cells and in stromal tissues. However, contrary to our expectations, positively stained cells for TSP-3 to 5 were rare in the cancer stromal area. This finding was similar to that in other types of cancer $(34,37,53)$. Another limitation of the study is that the detailed pathological roles of TPS-4 are unclear. In short, although over $40 \%$ of patients were judged as positive for TSP-4 expression despite its lack of expression in normal urothelial cells, no significant role of TSP-4 was determined. Based on previous reports in other malignancies (34-37), we speculate that TSP-4 might play significant roles in $\mathrm{BC}$, except in cell survival and angiogenesis. In addition, TSP-4 expressed in stromal cells might play important roles in malignant aggressiveness, though the methods used in this study cannot detect such expression. Therefore, we emphasize the need for further detailed studies using cancer cell lines, animal experiments, and clinicopathological data regarding the pathological roles of TSP-3 to 5 expression in BC.

In conclusion, the present study demonstrated that TSP-5 expression was significantly associated with malignant aggressiveness and survival via regulation of cancer cell proliferation and apoptosis. These results were not confirmed with the expression of TSP-3 and 4. Based on our results, we suggest that TSP-5 is a potential therapeutic target and useful predictive marker in patients with $\mathrm{BC}$.

\section{Conflicts of Interest}

None of the Authors have any conflicts of interest regarding this study.

\section{Authors' Contributions}

Study concept: YMiyata. Study design: YMiyata. Clinical data collection: KA, KM, TMatsuo, KO and YMochizuki, Immuno- histochemical analyses: JH, YM, KA, TMatsuda, YN, and Ymukae. Statistical analyses: YMiyata and TMatsuo. Manuscript preparation: JH, YMi, and KA. Manuscript editing: YMmochizuki. Manuscript review: HS. All Authors read and approved the final manuscript.

\section{Acknowledgements}

This study was supported in part by funds provided by KAKENHI (Grant-in-Aid for Exploratory Research) to Yasuyoshi Miyata (16K15690).

\section{References}

1 Witjes JA, Bruins HM, Cathomas R, Compérat EM, Cowan NC, Gakis G, Hernández V, Linares Espinós E, Lorch A, Neuzillet Y, Rouanne M, Thalmann GN, Veskimäe E, Ribal MJ and van der Heijden AG: European Association of Urology Guidelines on Muscle-invasive and Metastatic Bladder Cancer: Summary of the 2020 Guidelines. Eur Urol 79(1): 82-104, 2021. PMID: 32360052. DOI: 10.1016/j.eururo.2020.03.055

2 Miyata Y, Kanda S, Mitsunari K, Asai A and Sakai H: Heme oxygenase-1 expression is associated with tumor aggressiveness and outcomes in patients with bladder cancer: A correlation with smoking intensity. Transl Res 164(6): 468-476, 2014. PMID: 25063314. DOI: $10.1016 / \mathrm{j} . \operatorname{trsl} .2014 .06 .010$

3 Matsuo T, Miyata Y, Mitsunari K, Yasuda T, Ohba K and Sakai H: Pathological significance and prognostic implications of heme oxygenase 1 expression in non-muscle-invasive bladder cancer: Correlation with cell proliferation, angiogenesis, lymphangiogenesis and expression of VEGFs and COX-2. Oncol Lett 13(1): 275-280, 2017. PMID: 28123555. DOI: 10.3892/ol.2016.5416

4 Xiang M, Yuan W, Zhang W and Huang J: Expression of miR490-5p, miR-148a-3p and miR-608 in bladder cancer and their effects on the biological characteristics of bladder cancer cells. Oncol Lett 17(5): 4437-4442, 2019. PMID: 30988813. DOI: 10.3892/ol.2019.10143

5 Miyata $\mathrm{Y}$ and Sakai H: Predictive markers for the recurrence of nonmuscle invasive bladder cancer treated with intravesical therapy. Dis Markers 2015: 857416, 2015. PMID: 26681820. DOI: $10.1155 / 2015 / 857416$

6 Kim J, Jin P, Yang W and Kim WJ: Proteomic profiling of bladder cancer for precision medicine in the clinical setting: A review for the busy urologist. Investig Clin Urol 61(6): 539-554, 2020. PMID: 33135400. DOI: 10.4111/icu.20200317

7 Wu Y, Peng Y, Guan B, He A, Yang K, He S, Gong Y, Li X and Zhou L: P4HB: A novel diagnostic and prognostic biomarker for bladder carcinoma. Oncol Lett 21(2): 95, 2021. PMID: 33376528. DOI: 10.3892/ol.2020.12356

8 Sid B, Sartelet H, Bellon G, El Btaouri H, Rath G, Delorme N, Haye B and Martiny L: Thrombospondin 1: A multifunctional protein implicated in the regulation of tumor growth. Crit Rev Oncol Hematol 49(3): 245-258, 2004. PMID: 15036264. DOI: 10.1016/j.critrevonc.2003.09.009

9 Neff LS and Bradshaw AD: Cross your heart? Collagen crosslinks in cardiac health and disease. Cell Signal 79: 109889, 2021. PMID: 33347984. DOI: 10.1016/j.cellsig.2020.109889

10 Ramchandani D and Mittal V: Thrombospondin in tumor microenvironment. Adv Exp Med Biol 1272: 133-147, 2020. PMID: 32845506. DOI: 10.1007/978-3-030-48457-6_8 
11 Miyata Y, Koga S, Takehara K, Kanetake H and Kanda S: Expression of thrombospondin-derived $4 \mathrm{~N} 1 \mathrm{~K}$ peptide-containing proteins in renal cell carcinoma tissues is associated with a decrease in tumor growth and angiogenesis. Clin Cancer Res 9(5): 1734-1740, 2003. PMID: 12738728.

12 Miyata Y and Sakai H: Thrombospondin-1 in urological cancer: Pathological role, clinical significance, and therapeutic prospects. Int J Mol Sci 14(6): 12249-12272, 2013. PMID: 23749112. DOI: $10.3390 /$ ijms 140612249

13 Weng TY, Wang CY, Hung YH, Chen WC, Chen YL and Lai MD: Differential expression pattern of THBS1 and THBS2 in lung cancer: Clinical outcome and a systematic-analysis of microarray databases. PLoS One 11(8): e0161007, 2016. PMID: 27513329. DOI: 10.1371/journal.pone.0161007

14 Nakamura Y, Miyata Y, Takehara K, Asai A, Mitsunari K, Araki $\mathrm{K}$, Matsuo T, Ohba K and Sakai H: The pathological significance and prognostic roles of Thrombospondin-1, and -2, and 4N1Kpeptide in bladder cancer. Anticancer Res 39(5): 2317-2324, 2019. PMID: 31092423. DOI: 10.21873/anticanres.13348

15 Qi C, Lei L, Hu J, Wang G, Liu J and Ou S: Thrombospondin-1 is a prognostic biomarker and is correlated with tumor immune microenvironment in glioblastoma. Oncol Lett 21(1): 22, 2021. PMID: 33240428. DOI: 10.3892/ol.2020.12283

16 Kamijo H, Miyagaki T, Takahashi-Shishido N, Nakajima R, Oka $\mathrm{T}$, Suga H, Sugaya $M$ and Sato S: Thrombospondin-1 promotes tumor progression in cutaneous T-cell lymphoma via CD47. Leukemia 34(3): 845-856, 2020. PMID: 31712778. DOI: $10.1038 / \mathrm{s} 41375-019-0622-6$

17 Kashihara H, Shimada M, Yoshikawa K, Higashijima J, Tokunaga T, Nishi M, Takasu C and Ishikawa D: Correlation between Thrombospondin-1 expression in non-cancer tissue and gastric carcinogenesis. Anticancer Res 37(7): 3547-3552, 2017. PMID: 28668845. DOI: 10.21873 /anticanres.11724

18 Chatila K, Ren G, Xia Y, Huebener P, Bujak M and Frangogiannis NG: The role of the thrombospondins in healing myocardial infarcts. Cardiovasc Hematol Agents Med Chem 5(1): 21-27, 2007. PMID: 17266545. DOI: 10.2174/ 187152507779315813

19 Mustonen E, Ruskoaho H and Rysä J: Thrombospondins, potential drug targets for cardiovascular diseases. Basic Clin Pharmacol Toxicol 112(1): 4-12, 2013. PMID: 23074998. DOI: $10.1111 /$ bcpt.12026

20 Luo Y, Zeng G and Wu S: Identification of microenvironmentrelated prognostic genes in bladder cancer based on gene expression profile. Front Genet 10: 1187, 2019. PMID: 31824575. DOI: 10.3389/fgene.2019.01187

21 Helkin A, Maier KG and Gahtan V: Thrombospondin-1, -2 and -5 have differential effects on vascular smooth muscle cell physiology. Biochem Biophys Res Commun 464(4): 1022-1027, 2015. PMID: 26168731. DOI: 10.1016/j.bbrc.2015.07.044

22 Muqri F, Helkin A, Maier KG and Gahtan V: Thrombospondin5 and fluvastatin promote angiogenesis and are protective against endothelial cell apoptosis. J Cell Biochem 121(10): 4154-4165, 2020. PMID: 32348623. DOI: 10.1002/jcb.29686

23 Englund E, Bartoschek M, Reitsma B, Jacobsson L, EscuderoEsparza A, Orimo A, Leandersson K, Hagerling C, Aspberg A, Storm P, Okroj M, Mulder H, Jirström K, Pietras K and Blom AM: Cartilage oligomeric matrix protein contributes to the development and metastasis of breast cancer. Oncogene 35(43): 5585-5596, 2016. PMID: 27065333. DOI: 10.1038/onc.2016.98
24 Englund E, Canesin G, Papadakos KS, Vishnu N, Persson E, Reitsma B, Anand A, Jacobsson L, Helczynski L, Mulder H, Bjartell A and Blom AM: Cartilage oligomeric matrix protein promotes prostate cancer progression by enhancing invasion and disrupting intracellular calcium homeostasis. Oncotarget $8(58)$ : 98298-98311, 2017. PMID: 29228690. DOI: 10.18632/ oncotarget.21176

25 Mitsunari K, Miyata Y, Watanabe SI, Asai A, Yasuda T, Kanda $\mathrm{S}$ and Sakai H: Stromal expression of Fer suppresses tumor progression in renal cell carcinoma and is a predictor of survival. Oncol Lett 13(2): 834-840, 2017. PMID: 28356966. DOI: 10.3892/ol.2016.5481

26 Yuno T, Miyata Y, Matsuo T, Mukae Y, Otsubo A, Mistunari K, Ohba K, Suda T, Saito S and Sakai H: Relationship between stage-specific Embryonic Antigen-4 and anti-cancer effects of neoadjuvant hormonal therapy in prostate cancer. Anticancer Res 40(10): 5567-5575, 2020. PMID: 32988880. DOI: 10.21873/ anticanres.14569

27 Liao Q, Kleeff J, Xiao Y, Di Cesare PE, Korc M, Zimmermann A, Büchler MW and Friess H: COMP is selectively up-regulated in degenerating acinar cells in chronic pancreatitis and in chronic-pancreatitis-like lesions in pancreatic cancer. Scand J Gastroenterol 38(2): 207-215, 2003. PMID: 12678339. DOI: $10.1080 / 00365520310000717$

28 Han J, Chen M, Wang Y, Gong B, Zhuang T, Liang L and Qiao $\mathrm{H}$ : Identification of biomarkers based on differentially expressed genes in papillary thyroid carcinoma. Sci Rep 8(1): 9912, 2018. PMID: 29967488. DOI: 10.1038/s41598-018-28299-9

29 Liu TT, Liu XS, Zhang M, Liu XN, Zhu FX, Zhu FM, Ouyang SW, Li SB, Song CL, Sun HM, Lu S, Zhang Y, Lin J, Tang HM and Peng ZH: Cartilage oligomeric matrix protein is a prognostic factor and biomarker of colon cancer and promotes cell proliferation by activating the Akt pathway. J Cancer Res Clin Oncol 144(6): 1049-1063, 2018. PMID: 29560517. DOI: 10.1007/s00432-018-2626-4

30 Zhong W, Hou H, Liu T, Su S, Xi X, Liao Y, Xie R, Jin G, Liu X, Zhu L, Zhang H, Song X, Yang C, Sun T, Cao H and Wang $\mathrm{B}$ : Cartilage oligomeric matrix protein promotes epithelialmesenchymal transition by interacting with Transgelin in colorectal cancer. Theranostics 10(19): 8790-8806, 2020. PMID: 32754278. DOI: $10.7150 /$ thno. 44456

31 Nfonsam VN, Nfonsam LE, Chen D, Omesiete PN, Cruz A, Runyan RB and Jandova J: COMP gene coexpresses with EMT genes and is associated with poor survival in colon cancer patients. J Surg Res 233: 297-303, 2019. PMID: 30502262. DOI: $10.1016 /$ j.jss.2018.08.021

32 Maruta S, Miyata Y, Sagara Y, Kanda S, Iwata T, Watanabe SI, Sakai H, Hayashi $\mathrm{T}$ and Kanetake H: Expression of matrix metalloproteinase-10 in non-metastatic prostate cancer: Correlation with an imbalance in cell proliferation and apoptosis. Oncol Lett 1(3): 417-421, 2010. PMID: 22966318. DOI: 10.3892/ol_00000073

33 Król SK, Bębenek E, Sławińska-Brych A, DmoszyńskaGraniczka M, Boryczka S and Stepulak A: Synthetic Betulin derivatives inhibit growth of glioma cells in vitro. Anticancer Res 40(11): 6151-6158, 2020. PMID: 33109552. DOI: 10.21873/ anticanres. 14635

34 Kuroda K, Yashiro M, Sera T, Yamamoto Y, Kushitani Y, Sugimoto A, Kushiyama S, Nishimura S, Togano S, Okuno T, Tamura T, Toyokawa T, Tanaka H, Muguruma K and Ohira M: 
The clinicopathological significance of Thrombospondin-4 expression in the tumor microenvironment of gastric cancer. PLoS One 14(11): e0224727, 2019. PMID: 31703077. DOI: 10.1371/journal.pone.0224727

$35 \mathrm{Wu} \mathrm{H}$, Zhang G, Li Z, Ma J, Han X, Xiang T and Jiang X: Thrombospondin-4 expression as a prognostic marker in hepatocellular carcinoma. Gene 696: 219-224, 2019. PMID: 30802535. DOI: 10.1016/j.gene.2019.02.049

36 Guo D, Zhang D, Ren M, Lu G, Zhang X, He S and Li Y: THBS4 promotes HCC progression by regulating ITGB1 via FAK/PI3K/AKT pathway. FASEB J 34(8): 10668-10681, 2020. PMID: 32567740 . DOI: 10.1096/fj.202000043R

37 Shi Y, Sun L, Zhang R, Hu Y, Wu Y, Dong X, Dong D, Chen C, Geng Z, Li E and Fan Y: Thrombospondin 4/integrin $\alpha 2 / \mathrm{HSF} 1$ axis promotes proliferation and cancer stem-like traits of gallbladder cancer by enhancing reciprocal crosstalk between cancer-associated fibroblasts and tumor cells. J Exp Clin Cancer Res 40(1): 14, 2021. PMID: 33407730. DOI: 10.1186/s13046020-01812-7

38 Rahman MT, Muppala S, Wu J, Krukovets I, Solovjev D, Verbovetskiy D, Obiako C, Plow EF and Stenina-Adognravi O: Effects of thrombospondin-4 on pro-inflammatory phenotype differentiation and apoptosis in macrophages. Cell Death Dis 11(1): 53, 2020. PMID: 31974349. DOI: 10.1038/s41419-0202237-2

39 McCart Reed AE, Song S, Kutasovic JR, Reid LE, Valle JM, Vargas AC, Smart CE and Simpson PT: Thrombospondin-4 expression is activated during the stromal response to invasive breast cancer. Virchows Arch 463(4): 535-545, 2013. PMID: 23942617. DOI: $10.1007 / \mathrm{s} 00428-013-1468-3$

40 Greco SA, Chia J, Inglis KJ, Cozzi SJ, Ramsnes I, Buttenshaw RL, Spring KJ, Boyle GM, Worthley DL, Leggett BA and Whitehall VL: Thrombospondin-4 is a putative tumoursuppressor gene in colorectal cancer that exhibits age-related methylation. BMC Cancer 10: 494, 2010. PMID: 20846368. DOI: 10.1186/1471-2407-10-494

41 Su F, Zhao J, Qin S, Wang R, Li Y, Wang Q, Tan Y, Jin H, Zhu F, Ou Y, Cheng Z, Su W, Zhao F, Yang Y, Zhou Z, Zheng J, Li $\mathrm{Y}, \mathrm{Li} \mathrm{Z}$ and $\mathrm{Wu} \mathrm{Q}$ : Over-expression of Thrombospondin 4 correlates with loss of miR-142 and contributes to migration and vascular invasion of advanced hepatocellular carcinoma. Oncotarget 8(14): 23277-23288, 2017. PMID: 28177895. DOI: 10.18632/oncotarget.15054

42 Dalla-Torre CA, Yoshimoto M, Lee CH, Joshua AM, de Toledo SR, Petrilli AS, Andrade JA, Chilton-MacNeill S, Zielenska M and Squire JA: Effects of THBS3, SPARC and SPP1 expression on biological behavior and survival in patients with osteosarcoma. BMC Cancer 6: 237, 2006. PMID: 17022822. DOI: $10.1186 / 1471-2407-6-237$

43 Pan MR, Chang HC, Chuang LY and Hung WC: The nonsteroidal anti-inflammatory drug NS398 reactivates SPARC expression via promoter demethylation to attenuate invasiveness of lung cancer cells. Exp Biol Med (Maywood) 233(4): 456-462, 2008. PMID: 18367635. DOI: 10.3181/0709-RM-257
44 Tran PHL, Lee BJ and Tran TTD: Current studies of aspirin as an anticancer agent and strategies to strengthen its therapeutic application in cancer. Curr Pharm Des: 2020. PMID: 33138752. DOI: $10.2174 / 1381612826666201102101758$

45 Wu X, Luo X, Zhu Q, Zhang J, Liu Y, Luo H, Cheng Y and Xie $\mathrm{Z}$ : The roles of Thrombospondins in hemorrhagic stroke. Biomed Res Int 2017: 8403184, 2017. PMID: 29214179. DOI: $10.1155 / 2017 / 8403184$

46 Stenina-Adognravi O, Muppala $\mathrm{S}$ and Gajeton J: Thrombospondins and remodeling of the tumor microenvironment. Vessel Plus 2: 2018. PMID: 32984773. DOI: 10.20517/2574-1209.2018.40

47 Qabar AN, Bullock J, Matej L and Polverini P: Expression and characterization of novel thrombospondin 1 type I repeat fusion proteins. Biochem J 346 Pt 1: 147-153, 2000. PMID: 10657251

48 Horiguchi H, Jin L, Ruebel KH, Scheithauer BW and Lloyd RV: Regulation of VEGF-A, VEGFR-I, thrombospondin-1, -2, and 3 expression in a human pituitary cell line (HP75) by TGFbeta1, bFGF, and EGF. Endocrine 24(2): 141-146, 2004. PMID: 15347840. DOI: $10.1385 /$ ENDO:24:2:141

49 Muppala S, Frolova E, Xiao R, Krukovets I, Yoon S, Hoppe G, Vasanji A, Plow E and Stenina-Adognravi O: Proangiogenic properties of Thrombospondin-4. Arterioscler Thromb Vasc Biol 35(9): 1975-1986, 2015. PMID: 26139464. DOI: 10.1161/ ATVBAHA.115.305912

50 Stenina-Adognravi $\mathrm{O}$ and Plow EF: Thrombospondin-4 in tissue remodeling. Matrix Biol 75-76: 300-313, 2019. PMID: 29138119. DOI: $10.1016 /$ j.matbio.2017.11.006

51 Muppala S, Xiao R, Krukovets I, Verbovetsky D, Yendamuri R, Habib N, Raman P, Plow E and Stenina-Adognravi O: Thrombospondin-4 mediates TGF- $\beta$-induced angiogenesis. Oncogene 36(36): 5189-5198, 2017. PMID: 28481870. DOI: 10.1038/onc.2017.140

52 Kim MS, Choi HS, Wu M, Myung J, Kim EJ, Kim YS, Ro S, Ha SE, Bartlett A, Wei L, Ryu HS, Choi SC, Park WC, Kim KY and Lee MY: Potential Role of PDGFR $\beta$-Associated THBS4 in colorectal cancer development. Cancers (Basel) 12(9): 2020. PMID: 32899998. DOI: 10.3390/cancers12092533

53 Li F, Teng H, Liu M, Liu B, Zhang D, Xu Z, Wang Y and Zhou $\mathrm{H}$ : Prognostic value of immune-related genes in the tumor microenvironment of bladder cancer. Front Oncol 10: 1302, 2020. PMID: 32850407. DOI: 10.3389/fonc.2020.01302
Received February 5, 2021

Revised February 25, 2021

Accepted February 26, 2021 